\title{
Efficacy of low dose and ultra-low dose on the visibility of peri-implant fenestration and dehiscences: a computed tomography study
}

\author{
Ceren Aktuna-Belgin ${ }^{1, A, B, D, E, F, F}$, Gozde Serindere ${ }^{1, A, D, E, F}$, Huseyin Berkay Belgin ${ }^{2, A, B, C, D}$, Mehmet Serindere ${ }^{3, A, C, D, F}$, \\ Kaan Orhan ${ }^{4, A, C, D, E}$ \\ 'Department of Dentomaxillofacial Radiology, Faculty of Dentistry, Hatay Mustafa Kemal University, Hatay, Turkey \\ 2Private Dental Clinic, Turkey \\ ${ }^{3}$ Hatay Education and Research Hospital, Hatay, Turkey \\ ${ }^{4}$ Department of Dentomaxillofacial Radiology, Faculty of Dentistry, Ankara University, Ankara, Turkey
}

\section{Abstract}

Purpose: This study aimed to evaluate the visibility of peri-implant fenestration and dehiscences on computed tomography (CT) images taken with 2 different doses.

Material and methods: The defects were created on the apical of 6 implants randomly selected from 20 titanium implants placed in the ribs, and dehiscences were created on the cervical of 8 implants. No defects were created around 6 implants. Macroscopic analysis of the implanted ribs was accepted as the gold standard. From the samples, images were taken by using both ultra-low dose $(80 \mathrm{kVp}, 50 \mathrm{~mA}, 1.25 \mathrm{~mm}$ slice thickness) and low dose (100 kVp, $50 \mathrm{~mA}$, $1.25 \mathrm{~mm}$ slice thickness) protocols in CT. The images obtained were evaluated using a 5-point scale.

Results: No significant difference was found between the area under the receiver operating characteristic of ultra-low dose protocol and low dose protocol in both defects based on the Wilcoxon test $(p>0.05)$.

Conclusions: The ultra-low dose protocol could be applied by adhering to the "as low as reasonably achievable" principle in the diagnosis of peri-implant defects.

Key words: computed tomography, radiation dose, implant, fenestration, dehiscences.

\section{Introduction}

For dental implants to be considered clinically successful, should absent pain and tenderness during function, mobility, or abscesses. It is also stated that stable, rigid implants with a pocket depth of 2 to $6 \mathrm{~mm}$ can be considered successful [1]. When examined radiologically, vertical marginal bone loss at the peri-implant surface should not exceed 1-2 $\mathrm{mm}$ in the first year after implant loading and $0.2 \mathrm{~mm}$ in later years [2]. Another factor that determines the success of dental implants is whether there is sufficient bone volume in the area where the implant is placed [3]. Insufficient bone in the buccolingual direction can lead to bone fenestrations and dehiscences formation, which compromise the long-term prognosis of dental implants [4]. Dehiscences is a buccal or facial alveolar bonelimited defect that exposes the roots of the dental implant or teeth from the alveolar crest to the apical. Fenestration is a window-shaped defect of $3 \mathrm{~mm}$ or more when the implant or tooth root is covered with gingival tissues rather than bone [5].

The determination of alveolar bone defects plays a role in determining the need for grafts before surgical procedures [6]. However, insufficient bone thickness can lead to negative

Correspondence address:

Dr. Ceren Aktuna-Belgin, Department of Dentomaxillofacial Radiology, Faculty of Dentistry, Hatay Mustafa Kemal University, Hatay, Turkey, e-mail: dtcaktuna@gmail.com

Authors' contribution:

A Study design · B Data collection - C Statistical analysis · D Data interpretation · E Manuscript preparation · F Literature search · G Funds collection 
consequences such as inaccurate implant placement during surgery, inflammatory biofilm, and occlusion errors. Moreover, it can lead to gingival recession, loss of alveolar bone, failing osseointegration, and implant failures $[3,5,7,8]$.

The most common method for assessing marginal bone loss is conventional periapical radiography [1]. In implants, bone loss after the 1st placement occurs mostly on the facial or buccal side of the implant because the bone is thinner in these areas. However, periapical radiographs can detect bone loss in the mesial or distal. Because the buccal or facial area is superposed on 2-dimensional periapical radiographs, it is inadequate to show early bone destruction $[9,10]$. In computed tomography (CT), a 3-dimensional evaluation without superposition can be detected buccolingual or mesiodistal alveolar bone destruction [11]. Although CT has been shown to yield accurate and reliable assessments for orthodontic and maxillofacial applications, its effective dose is much higher than that of conventional radiography or cone beam CT (CBCT) [12].

As a result of technological advances in the field of dentistry, both digital imaging techniques and 3-dimensional imaging techniques have been introduced in the routine. Especially since the introduction of CBCT devices, the interest in $3 \mathrm{D}$ imaging has increased rapidly in the last decade $[13,14]$.

Although CBCT is routinely using for implant planning, CT is also used in some instances where CBCT is not available. Moreover, with the introduction of modern multiple-detector computed tomography (MDCT) technology and continuing technological developments, especially iterative reconstruction algorithms, low dose CT examinations are currently employed, such as in lung and maxillofacial imaging. Various $\mathrm{kV}$ and $\mathrm{mAs}$ values have been used in MDCT with different estimated effective doses. A recent study, aimed at determining the distribution of effective dose in paranasal sinus imaging associated with a single low dose CT examination concluded that acceptable CT screening can be accomplished at an overall average computer tomography dose index (CTDI) of approximately $2.9 \mathrm{mGy}$ [15].

Also, the ALARA (as low as reasonably achievable) principle, which is an underlined view in radiation safety, emphasizes that the radiation dose given to living structures is the lowest possible value without affecting diagnosis and treatment. Following this principle, the most appropriate dose protocol should be selected based on the reliability and accuracy of the clinical diagnostic result and radiation dose [16].

Moreover, no extensive data on the use of low dose CT are available, and further studies are needed to assess the usefulness of this technique for maxillofacial and dental imaging.

The purpose of this study was to evaluate the visibility of peri-implant fenestrations and dehiscences for dental implant exposures from ultra-low dose and low dose CT set-ups. The null hypothesis indicated that there was no significant difference in the detection of peri-implant defects at the 2 different dose set-ups on the detection of peri-implant defects.

\section{Material and methods}

\section{Study design}

The sample size was calculated with the GPower 3.1 software (http://www.gpower.hhu.de/); the total mean of 2 groups compared based on Student's $t$-test with the power of $80 \%$ and 0.05 type 1 error was found to be at least 13 patients.

Based on the study of de-Azevedo-Vaz et al. [4], soft tissue-free ribs were used to represent the alveolar bone. Fresh beef ribs were obtained from a local butcher from slaughtered animals. A total of 20 dental implants (NTA, Pilatus Swiss Dental, Egolzwil, Switzerland) were used in diameter range, 3.7-5.1 $\mathrm{mm}$; length range, $8.5-10 \mathrm{~mm}$. After placement of 20 titanium implants, similarly to the literature [17], 3-mm diameter fenestrations and dehiscences were created on the rib. In the apical of 6 randomly selected implants, fenestrations were formed in elliptical form $10 \mathrm{~mm}$ apical to the upper edge of the rib. In the cervical of 8 implants, half-elliptical form dehiscences were formed on the alveolar crest. No defects were created around the other 6 implants, and they were evaluated as a control group.

\section{Radiographic imaging}

CTs were taken after the ribs were placed in a water-filled container to resemble soft-tissue attenuation. CT images were performed with a 256-slice CT scanner (Revolution CT; GE Healthcare). To evaluate the visibility of the created defects in 2 different doses, the samples were firstly taken with the ultra-low dose protocol $(80 \mathrm{kVp}, 50 \mathrm{~mA}, 1.25 \mathrm{~mm}$ slice thickness). Then the same samples were scanned with the low dose protocol $(100 \mathrm{kVp}, 50 \mathrm{~mA}, 1.25 \mathrm{~mm}$ slice thickness). The size-specific dose estimates in CT (SSDE) of the ultra-low dose protocol and low dose protocol were $6.66 \mathrm{mGy}$ and $12.14 \mathrm{mGy}$, respectively. The dose length protocol (DLP) of the ultra-low dose and low dose protocol was $77.32 \mathrm{mGy} \mathrm{cm}$ and $171.57 \mathrm{mGy} \mathrm{cm}$, respectively. The CTDI of the ultra-low dose protocol and low dose protocol was $5.95 \mathrm{mGy}$ and $10.90 \mathrm{mGy}$, respectively (Figure 1).

\section{Sample analysis}

CT images were evaluated by 2 oral radiologists (C.A.B. and G.S., 5 and 6 years of clinical experience), 1 radiologist (M.S., 6 years of clinical experience), who did not know whether there were defects. A 5-point scale was used to evaluate the images ( 1 - defect absent; 2 - defect probably absent; 3 - uncertain; 4 - defect probably present; 5 - defect present). Macroscopic analysis of the 

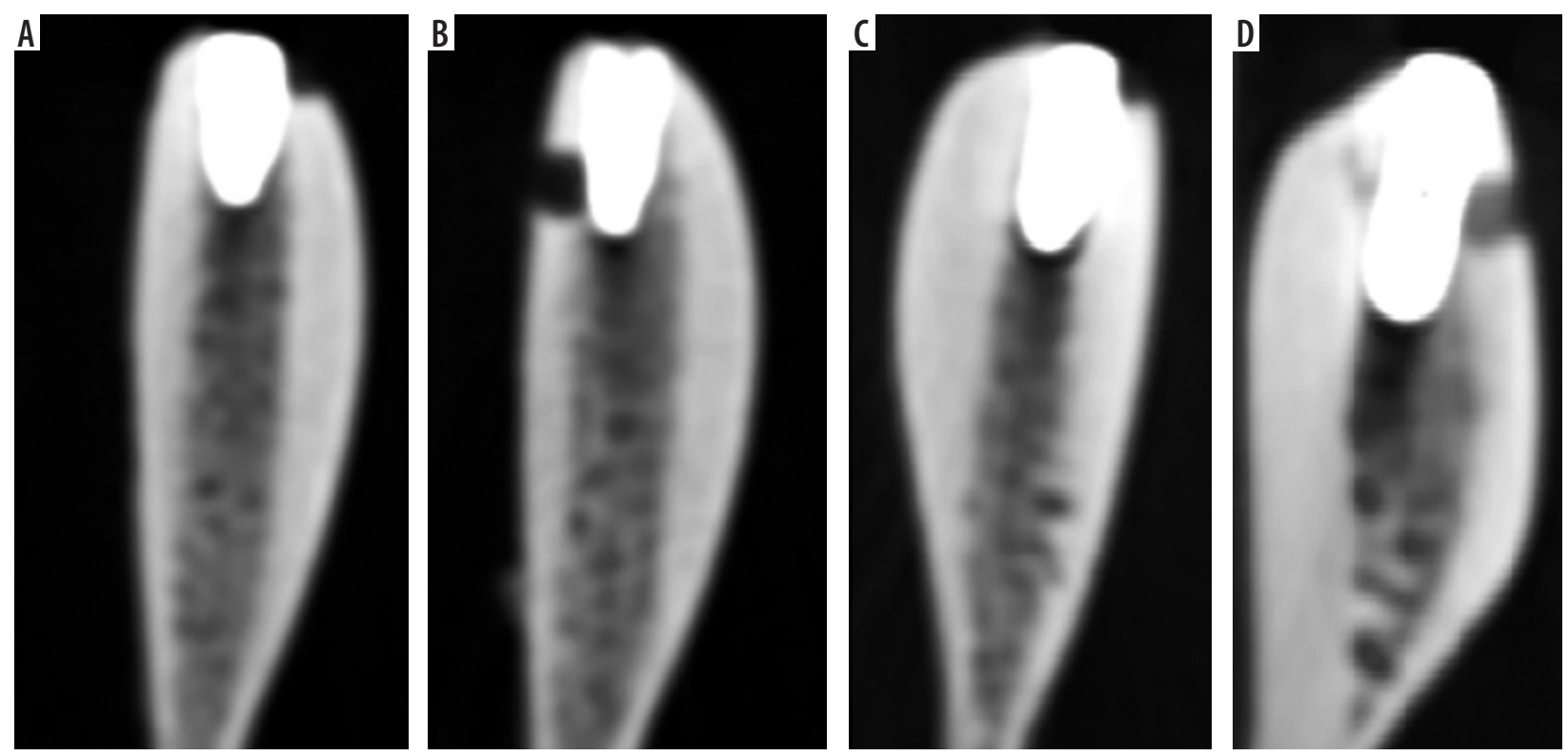

Figure 1. Sagittal slice on computed tomography images of titanium implant with simulated peri-implant defects with 2 different dose protocols. A) Dehiscences on low dose, B) fenestration on low dose, C) dehiscences on the ultra-low dose, D) fenestration on the ultra-low dose

implanted ribs was accepted as the gold standard (defect present or absent) [4]. Evaluations were made with a RadiAnt Dicom Viewer (2020.1, Medixant, Poznan, Poland). Observers were allowed to view the images in 3 planes (sagittal, axial, coronal), change the contrast or brightness of the images, and use the rotate or zoom tool.

\section{Statistical analysis}

Kappa's $\alpha$ was obtained regarding compatibility among the observers' agreement on the correct diagnosis of the defect types between the 2 protocols using SPSS 26.0. (SPSS Inc., Chicago, IL). Based on Landis and Koch [18], low agreement -0.40 ; moderate agreement $-0.40-0.59$; good agreement - 0.60-0.74; and excellent agreement - 0.75-1.00 were considered. The areas under receiver operating characteristic (ROC) curves (Az values) for the 2 protocols for each observer were computed from the ROC-curve and compared with the Wilcoxon-test at a $5 \%$ significance level. Also, the sensitivity (the correct identification of a defect presence), specificity (the correct identification of a defect absence), accuracy (proportion of correctness), positive predictive value (PPV: the probability that a positive evaluation is a true-positive result), and negative predictive value (NPV: the probability that a negative evaluation is a true-negative result) were calculated for each protocol. In the study, assessment of the compatibility of diagnosis between observers revealed a group of individuals in the group of people who have the case group and in the control group.

\section{Results}

The average and standard deviations of the kappa $\alpha$ values are shown in Table 1. The inter-observer agreement of the ultra-low dose protocol was good, and the inter-observer agreement of the low dose protocol was excellent.

The Az values and $p$-values for the diagnosis of periimplant defects are shown in Table 2. The diagnosis of observers was equal at both dose protocol for fenestration and dehiscence diagnosis without a significant difference. Also, there was also no significant difference between Az values of the ultra-low dose and low dose protocol in both defects.

Table 1. Values of kappa's $\alpha$ for inter-observer agreement

\begin{tabular}{|l|c|c|}
\hline \multicolumn{1}{|l|}{} & Fenestration, mean (SD) & Dehiscences, mean (SD) \\
\hline Ultra-low dose & $0.64(0.03)$ & $0.62(0.11)$ \\
\hline Low dose & $0.72(0.17)$ & $0.75(0.14)$ \\
\hline
\end{tabular}

Table 2. Value of the area under the receiver operating characteristic (ROC) curve (Az values) and $p$-value for each observer and protocols

\begin{tabular}{|l|l|c|c|}
\hline \multicolumn{2}{|c|}{} & $\begin{array}{c}\text { Ultra-low dose } \\
\text { Az values ( } p \text {-value) }\end{array}$ & $\begin{array}{c}\text { Low dose } \\
\text { Az values ( } p \text {-value) }\end{array}$ \\
\hline \multirow{5}{*}{ Fenestration } & Obs 1 & $1(0.004)$ & $1(0.004)$ \\
\cline { 2 - 5 } & Obs 2 & $1(0.004)$ & $1(0.004)$ \\
\cline { 2 - 5 } & Obs 3 & $1(0.004)$ & $1(0.004)$ \\
\cline { 2 - 5 } & Mean (SD) & 1 & 1 \\
\cline { 2 - 5 } & $p$-value & & 1.00 \\
\hline \multirow{5}{*}{ Dehiscences } & Obs 1 & $1(0.004)$ & $1(0.002)$ \\
\cline { 2 - 5 } & Obs 2 & $0.969(0.004)$ & $1(0.002)$ \\
\cline { 2 - 5 } & Obs 3 & $0.958(0.005)$ & $0.937(007)$ \\
\cline { 2 - 5 } & Mean (SD) & $0.975 \pm 0.02$ & $0.979 \pm 0.03$ \\
\cline { 2 - 4 } & $p$-value & \multicolumn{2}{|c}{0.15} \\
\hline
\end{tabular}

Obs - observer 
Table 3. Diagnostic values for fenestration and dehiscences using the tested protocols

\begin{tabular}{|l|l|c|c|}
\hline \multicolumn{2}{|l|}{ Parameter } & Ultra-low dose & Low dose \\
\hline \multirow{5}{*}{ Fenestration } & Sensitivity & 1 & 0.7 \\
\cline { 2 - 4 } & Specificity & 1 & 1 \\
\cline { 2 - 4 } & PPV & 1 & 1 \\
\cline { 2 - 4 } & NPV & 1 & 1 \\
\cline { 2 - 4 } & Accuracy & 1 & 0.85 \\
\hline \multirow{5}{*}{ Dehiscences } & Sensitivity & 1 & 0.91 \\
\cline { 2 - 4 } & Specificity & 1 & 0.62 \\
\cline { 2 - 4 } & PPV & 1 & 1 \\
\cline { 2 - 4 } & NPV & 1 & 1 \\
\cline { 2 - 4 } & Accuracy & 1 & 0.76 \\
\hline
\end{tabular}

PPV - positive predictive value, NPV - negative predictive value

Of all implants, 6 had fenestrations, 8 had dehiscences, and 6 had no defect. The sensitivity (the correct identification of a defect presence), specificity (the correct identification of a defect absence), accuracy (proportion of correctness), PPV, and NPV for the studied protocols are shown in Table 3. Comparison of both defects showed that values were higher for fenestrations than for dehiscences, but they were similar across protocols.

\section{Discussion}

Peri-implantitis is defects that can develop due to poor oral hygiene, history of periodontitis, bacteria, systemic diseases such as diabetes, smoking, or technical issues such as implant surface, type, structure, and osseointegration degree $[19,20]$. Peri-implant defects such as fenestrations and dehiscences are factors that decrease the success of the implant [1]. In addition to decreasing implant success, undiagnosed alveolar bone defects can also lead to a relapse of orthodontic treatment [21] or gingival recession that will result in an unaesthetic ending of orthodontic treatment [22]. Also, cases of peri-implantitis associated with endodontic problems have been reported in the literature [23].

Jeffcoat et al. [24] reported that 2-dimensional imaging is strongly affected by vertical and horizontal angulation errors during film exposure. Therefore, 3-dimensional imaging is recommended for the visualization of periodontal defects [25]. Radiation can cause carcinogenesis genetic damage to irradiated tissues [26]. Radiation is known to cause DNA damage with a low probability of ionization effect [27]. Therefore, radiography should be taken with the lowest radiation dose that will not affect the diagnosis and treatment, without damaging the biological structure in the area of interest [28].

In the present study, the effectiveness of the 2 different dose protocols invisibility of peri-implant defects were compared. As a result of this study, it was observed that there was no statistically significant difference between ultra-low dose and low dose protocols. Although in the literature there are studies visibility of peri-implant defects according to voxel size, the field of view, and the use of various algorithms and filters, there are few articles about dose-related [6,17,29-31]. Values from Misch et al. [30] (47.7 mA, $120 \mathrm{kVp}$ ) and Pinsky et al. [31] (98 mA, $120 \mathrm{kVp})$ were used as the scan protocol for the detection of fenestrations and dehiscences. In both studies, it was stated that artificially created fenestrations and dehiscences were identified and the measurement accuracy was high. In our study, 2 different scan protocols, $50 \mathrm{~mA} 100 \mathrm{kVp}$ and $50 \mathrm{~mA}$ $80 \mathrm{kVp}$, were used. Although a lower rate of $\mathrm{mA}$ and $\mathrm{kVp}$ was used than the work of both Misch et al. [30] and Pinsky et al. [31], defects were detected and there was no statistically significant difference between them. Bayrak et al. [17] evaluated the visibility of peri-implant defects with the metal artifact reduction algorithm and the adaptive image noise optimizer filter. As a result, they stated that the use of both filters together would be useful in detecting defects. In another study [29], 2 different voxel sizes $\left(0.2 \mathrm{~mm}^{3}\right.$ and $\left.0.12 \mathrm{~mm}^{3}\right)$, and two different scan modes $\left(180^{\circ}\right.$ and $\left.360^{\circ}\right)$, were used to detect defects. It was stated that the voxel size did not affect the visibility of defects, but dehiscences were seen more clearly with a $360^{\circ}$ scan mode. Kamburoglu et al. [6] investigated the effectiveness of the field of view in detecting peri-implant defects and stated that there was no relationship between the field of view and defect visibility. Interobserver agreement was good to excellent for the 2 different doses protocols in the present study. In this study, the inter-observer agreement of the ultra-low dose protocol was good, and the inter-observer agreement of the low dose protocol was excellent. However, de-Azevedo-Vaz et al. [4] found that inter-observer agreement was moderate to excellent in their study. The reason for this may be that the imaging techniques used in both studies are different.

\section{Limitations of the study}

This study has some limitations. The first is that the number of samples is low. It is thought that studies carried out on larger samples will yield more realistic results. Another is that the bone structure in which defects are formed did not belong to human bone. Although it is supported by the literature that cow ribs are similar to the human mandible bone in terms of bone density and cortical-cancellous bone relationship [32-34], the ribs do not mimic the human bone. Another limitation is the use of implants of different sizes and diameters. Whether different sizes and diameters of implants will change the result of the study may be the subject of another study.

\section{Conclusions}

In this study, it was found that there was no difference between the ultra-low dose protocol and the low dose protocol in imaging bone defects. In the radiological exami- 
nation of cases with peri-implantitis defects, it is thought that a lower dose protocol can be applied to patients following the ALARA principle.

\section{Conflict of interest}

The authors report no conflict of interest.

\section{References}

1. Misch CE, Perel ML, Wang HL, et al. Implant success, survival, and failure: the International Congress of Oral Implantologists (ICOI) Pisa Consensus Conference. Implant Dent 2008; 17: 5-15.

2. Smith DE, Zarb GA. Criteria for success of osseointegrated endosseous implants. J Prosthet Dent 1989; 62: 567-572.

3. Blanco J, Alonso A, Sanz M. Long-term results and survival rate of implants treated with guided bone regeneration: a 5-year case series prospective study. Clin Oral Impl Res 2005; 16: 294-301.

4. de-Azevedo-Vaz SL, Peyneau PD, Ramirez-Sotelo LR, et al. Efficacy of a cone-beam computed tomography metal artifact reduction algorithm for the detection of periimplant fenestrations and dehiscences. Oral Surg Oral Med Oral Pathol Oral Radiol 2016; 121: 550-556.

5. Leung CC, Palomo L, Griffith R, et al. Accuracy and reliability of conebeam computed tomography for measuring alveolar bone height and detecting bony dehiscences and fenestrations. Am J Orthod Dentofacial Orthop 2010; 137: 109-119.

6. Kamburoğlu K, Murat S, Kılıç C, et al. Accuracy of CBCT images in the assessment of buccal marginal alveolar peri-implant defects: effect of field of view. Dentomaxillofac Radiol 2014; 43: 20130332.

7. Mengel R, Kruse B, Flores-de-Jacoby L. Digital volume tomography in the diagnosis of peri-implant defects: an in vitro study on native pig mandibles. J Periodontol 2006; 77: 1234-1241.

8. Sancho-Puchades M, Hammerle CH, Benic GI. In vitro assessment of artifacts induced by titanium, titanium-zirconium, and zirconium dioxide implants in cone-beam computed tomography. Clin Oral Implants Res 2015; 26: 1222-1228.

9. Kamburoglu K, Gulsahi A, Genç Y, et al. A comparison of peripheral marginal bone loss at dental implants measured with conventional intraoral film and digitized radiographs. J Oral Implantol 2012; 38 : 211-219.

10. Schliephake H, Wichmann M, Donnerstag F, et al. Imaging of periimplant bone levels of implants with buccal bone defects. Clin Oral Implants Res 2003; 14: 193-200.

11. Pompa V, Galasso S, Cassetta M, et al. A comparative study of Magnetic Resonance (MR) and Computed Tomography (CT) in the pre-implant evaluation. Ann Stomatol (Roma) 2010; 1: 33-38.

12. Oz U, Orhan K, Abe N. Comparison of linear and angular measurements using two-dimensional conventional methods and three-dimensional cone beam CT images reconstructed from a volumetric rendering program in vivo. Dentomaxillofac Radiol 2011; 40: 492-500.

13. Shah N, Bansal N, Logani A. Recent advances in imaging technologies in dentistry. World J Radiol 2014; 6: 794-807.

14. Suomalainen A, Pakbaznejad Esmaeili E, Robinson S. Dentomaxillofacial imaging with panoramic views and cone beam CT. Insights Imaging 2015; 6: 1-16.

15. Hoxworth JM, Lal D, Fletcher GP, et al. Radiation dose reduction in paranasal sinus CT using model-based iterative reconstruction. AJNR Am J Neuroradiol 2014; 35: 644-649.
16. Kaplan DJ, Patel JN, Liporace FA, et al. Intraoperative radiation safety in orthopaedics: a review of the ALARA (as low as reasonably achievable) principle. Patient Saf Surg 2016; 10: 27.

17. Bayrak S, Orhan K, Kursun Çakmak ES, et al. Evaluation of a metal artifact reduction algorithm and an optimization filter in the estimation of peri-implant dehiscence defects by using cone beam computed tomography: an in-vitro study. Oral Surg Oral Med Oral Pathol Oral Radiol 2020; 130: 209-216.

18. Landis JR, Koch GC. The measurement of observer agreement for categorical data. Biometrics. 19 Am J Orthod Dentofacial Orthop 2006; 130: 476-484.

19. Lindhe J, Meyle J, Group D of European Workshop on Periodontology. Peri-implant diseases: consensus report of the sixth European workshop on periodontology. J Clin Periodontol 2008; 35: 282-285.

20. Smeets R, Henningsen A, Jung O, et al. Definition, etiology, prevention and treatment of peri-implantitis - a review. Head Face Med 2014; 10 : 34 .

21. Rothe LE, Bollen AM, Little RM, et al. Trabecular and cortical bone as risk factors for orthodontic relapse. Am J Orthod Dentofacial Orthop 2006; 130: 476-484.

22. Yared KF, Zenobio EG, Pacheco W. Periodontal status of mandibular central incisors after orthodontic proclination in adults. Am J Orthod Dentofacial Orthop 2006; 130: 6.e1-8.

23. Daubert D, Black RM, Chrepa V, et al. Endodontic peri-implant defects: a new disease entity. J Endod 2020; 46: 444-448.

24. Jeffcoat MK, Wang IC, Reddy MS. Radiographic diagnosis in periodontics. Periodontol 2000 1995; 7: 54-68.

25. Noujeim M, Prihoda T, Langlais R, et al. Evaluation of high-resolution cone beam computed tomography in the detection of simulated interradicular bone lesions. Dentomaxillofac Radiol 2009; 38 : 156-162.

26. Garib DG, Calil LR, Leal CR, et al. Is there a consensus for CBCT use in orthodontics? Dental Press J Orthod 2014; 19: 136-149.

27. Ludlow JB, Timothy R, Walker C, et al. Effective dose of dental CBCT-a meta-analysis of published data and additional data for nine CBCT units. Dentomaxillofac Radiol 2015; 44: 20140197.

28. American Academy of Oral and Maxillofacial Radiology. Clinical recommendations regarding use of cone beam computed tomography in orthodontics. [corrected]. Position statement by the American Academy of Oral and Maxillofacial Radiology. Oral Surg Oral Med Oral Pathol Oral Radiol 2013; 116: 238-257.

29. de-Azevedo-Vaz SL, Vasconcelos Kde F, Neves FS, et al. Detection of peri implant fenestration and dehiscences with the use of two scan modes and the smallest voxel sizes of a cone-beam computed tomography device. Oral Surg Oral Med Oral Pathol Oral Radiol 2013; 115: 121-127.

30. Misch KA, Yi ES, Sarment DP. Accuracy of cone beam computed tomography for periodontal defect measurements. J Periodontol 2006; 77: 1261-1266. 
31. Pinsky HM, Dyda S, Pinsky RW, et al. Accuracy of three-dimensional measurements using cone-beam CT. Dentomaxillofac Radiol 2006; 35: 410-416.

32. May KB, Edge MJ, Russell MM, et al. The precision of fit at the implant prosthodontic interface. J Prosthet Dent 1997; 77: 497-502.

33. Chiodo TA, Ziccardi VB, Janal M, et al. Failure strength of 2.0 locking versus 2.0 conventional Synthes mandibular plates: a laboratory model. Oral Maxillofac Surg 2006; 64: 1475-1479.

34. Choi BH, Huh JY, Suh CH, et al. An in vitro evaluation of miniplate fixation techniques for fractures of the atrophic edentulous mandible. Int J Oral Maxillofac Surg 2005; 34: 174-177. 